\title{
SERUM B12 DEFICIENCY;
} NEUROPSYCHIATRIC MANIFESTATIONS

Dr. Qamar-un-Nisa, Dr. Muhammad Tanveer Alam, Dr. Harilal Khiemani, Dr. Ayesha Chandna, Dr. Muhammad Wasay

ABSTRACT... Objective: To determine the frequency of various neurological and psychiatric symptoms associated with B12 deficiency. Study design: Observational and descriptive study. Place and duration of study: The study was conducted on patients presenting to the neurology clinics and inpatients who were admitted through the emergency department at Aga khan University Hospital Karachi, from 1st July 2011 to March 2012. Subjects and methods: 150 patients of either gender and age more than 18 years. Detailed history was taken from all the patients with special regard to anemia. Inclusion criteria were that patients above 18 years of age of either sex with low serum B12 levels. Patients having any type of neurological tumors, major ischemic stroke, major intracranial haemorrhage, patients on neuroleptic medications and any evidence of toxin induced neuropathy were excluded. All patients underwent for specific investigation complete blood count, serum B12 levels (derived by Radio Assay method (RIA). Further investigations Nerve Conduction Studies / Electromyography and CT Scan / Magnetic resonance Imaging of the Brain / spinal cord were carried out as and when required, depending on the clinical scenario. Results: There were 69 (46\%) males and $81(54 \%)$ females. Female to male ratio was 1:0.8. The age ranged between 18 to 90 years with the mean age of $45.7+5.6$. Out of 150 patients 107 patients $(71.3 \%)$ had severe vitamin B 12 deficiency while $43(28.7 \%)$ had mild to moderate deficiency. Neurological manifestations which were observed included; Memory was impaired in $13(8.7 \%)$ of the patients. Dysarthia, along with other cerebellar signs (Nystagmus and dysdiadochokinesia) was present in 9 patients (6\%). 4 patients (2.7\%) sought medical attention regarding their abnormal gait and their neurological examination revealed extensor planter responses and hyperreflexia. Cranial nerve examination revealed Anosmia in $2(1.3 \%)$ and upper motor neuron facial weakness in $1(0.66 \%)$ patients. Motor weakness was found in 2 patients (1. 3\%), and spasticity in $2(1.3 \%)$ patients. 5 patients $(3.3 \%)$ had sensory complains and were found to have impaired pain and touch perception. Proximal muscle weakness in one patient while another patient had asymmetric muscle weakness and rest of the patients had normal muscle power. None of the patient had autonomic dysfunction. In six neuropsychiatric problems were commonly seen in vitamin B 12 deficient people. In these patients depression was observed in 16\%, agitation $12.7 \%$, memory impairment $8.7 \%$, cerebellar signs and dysarthria $6 \%$, sensory symptoms $3.3 \%$ and gait abnormality and hyperreflexia and extensor planters $2.7 \%$. Conclusions: Various Neuropsychiatric problems are associated with vitamin B 12 deficiency and the most common Neuropsychiatric illness found in these patients were depression, agitation, and memory impairment.

Key words: Cobalamin, B12, deficiency, neuropsychiatric manifestations.

Article Citation

Qamar-un-Nisa, Alam MT, Kheimani H, Chandna A, Wasay M. Serum B12 Deficiency; Neuropsychiatric manifestations. Professional Med J Oct 2013;20(5): 688-693.

\section{INTRODUCTION}

Vitamin B12 deficiency is one of the few nutritional disorders encountered by neurologist. B12 deficiency is a common problem that affects general population especially the elderly ${ }^{1}$. Vitamin deficiencies are relatively uncommon in the Western world, it is estimated that $10 \%$ to $15 \%$ of individuals over the age of 60 years may suffer from B12 deficiency and it is assumed that atrophic gastritis type B, which may afflict $20 \%$ to $50 \%$ of the elderly, may lead to decrease absorption of B12 and folate ${ }^{2,3}$.

Neurologic sequelae from vitamin B12 deficiency include paresthesias, peripheral neuropathy and demyelination of the corticospinal tract and dorsal columns (subacute combined systems disease) ${ }^{4}$. Vitamin B12 deficiency also has been linked to psychiatric disorders, including impaired memory, depression, dementia and rarely, psychosis. Atypical presentations are also observed like anosmia, impotence and personality changes ${ }^{5}$. B12 is reported to be a reversible cause for orthostatic hypotension. Similar to folic acid deficiency, vitamin B12 deficiency produces hyperhomocysteinemia, which is an independent risk factor for atherosclerotic disease ${ }^{6}$. In this way B12 deficiency can indirectly lead to 
increased risk of cerebrovascular disease ${ }^{7}$. B12 deficiency, if identified early in the course and replaced, will result in reversal of the neurological symptoms ${ }^{8}$.

The present study is designed to identify various neurological and psychiatric symptoms associated with B12 deficiency in neurology outpatient clinic.

\section{MATERIAL AND METHODS}

The study was conducted on patients presenting to the neurology clinics and inpatients who were admitted through the emergency department at Aga khan University Hospital Karachi, from 1st July 2011 to March 2012. This study consisted of 150 cases. Detailed history was taken from all the patients with special regard to anemia. Inclusion criteria were that patients above 18 years of age of either sex with the having low serum B12 levels. Patients having any type of neurological tumors, major ischemic stroke, major intracranial haemorrhage, patients on neuroleptic medications and any evidence of toxin induced neuropathy were excluded.

Patients was diagnosed on basis of complete blood count, serum B12 levels (derived by Radio Assay method (RIA), laboratory reference range 200-900 pico grams/ ml) Levels upto150 picograms were classified as severely deficient, while levels from 151200 pico grams were considered to be mild to moderately deficient. Further investigations Nerve Conduction Studies / Electromyography and CT Scan / Magnetic resonance Imaging of the Brain / spinal cord were carried out as and when required, depending on the clinical scenario.

\section{RESULTS}

150 patients were enrolled with vitamin B12 deficiency. There were $69(46 \%)$ males and $81(54 \%)$ females. Female to male ratio was 1:0.8. The age ranged between 18 to 90 years with the mean age of $45.7+5.6$. Out of 150,12 patients (8\%) were less than 25 years of age, another 25 (16.7\%) were found to be between 25 to 39 years of age. 64 patients $(42.7 \%)$ belonged to the age group of 40 to 59 years of age. 49 patients (32.7\%)were. above the age of 60 years (Table-l). Out of 150 patients, $140(93.3 \%)$ belonged to non-vegetarian group and were Muslim by religion, while $10(6.7 \%)$ of studied population were found to be vegetarian and they were all non-Muslim. Out of 150 patients 107 patients $(71.3 \%$ ) had severe vitamin B 12 deficiency while $43(28.7 \%)$ had mild to moderate deficiency (Table-I).

\begin{tabular}{|c|c|c|}
\hline Variable & No. of patients & \%age \\
\hline \multicolumn{3}{|l|}{ Gender } \\
\hline Male & 69 & $46 \%$ \\
\hline Female & 81 & $54 \%$ \\
\hline \multicolumn{3}{|l|}{ Age } \\
\hline$<25$ years & 12 & $8 \%$ \\
\hline 25 to 39 years & 25 & $16.7 \%$ \\
\hline 40 to 49 years & 64 & $42.7 \%$ \\
\hline$>60$ years & 49 & $32.7 \%$ \\
\hline \multicolumn{3}{|l|}{ Diet } \\
\hline Vegetation & 10 & $6.7 \%$ \\
\hline Non vegetation & 140 & $93.3 \%$ \\
\hline \multicolumn{3}{|c|}{ Mild to moderate and severe disease } \\
\hline Mild to moderate & 43 & $28.7 \%$ \\
\hline Severe & 107 & $71.3 \%$ \\
\hline
\end{tabular}

In the studied population, most of the patients, i.e. 107 (71.3\%) had severe B12 deficiency .Out of 107 patient, most of them (43 patients, $40.18 \%$ ) belonged to the age group between $40-59$ years. 43 patients (28.7\%) had mild to moderately low levels. Out of 43 patients suffering from mild to moderate disease 21 patients (48.8\%) also affected the same age group. 
Our study showed that Psychiatric symptoms were found in 46 patients (30.7\%) Among them, depression was detected in $24(16 \%)$ patients while 19 patients (12.7\%) showed agitated behavior. Only 3 patients (2\%) had labile mood. Neurological manifestations which were observed included; Memory was impaired in $13(8.7 \%)$ of the patients, out of them, 10 patients (76.92\%) showed mildly low score on Mini mental state examination (mild dementia), while 3 patients showed no clinical sign of cognitive impairment. Dysarthia, along with other cerebellar signs (Nystagmus and dysdiadochokinesia) was present in 9 patients (6\%). 4 patients (2.7\%) sought medical attention regarding their abnormal gait and their neurological examination revealed extensor planter responses and hyperreflexia. Cranial nerve examination revealed Anosmia in $2(1.3 \%)$ and upper motor neuron facial weakness in $1(0.66 \%)$ patients. Motor weakness was found in 2 patients (1. 3\%), and Spasticity in $2(1.3 \%)$ patients. 5 patients $(3.3 \%)$ had sensory complains and were found to have impaired pain and touch perception. Proximal muscle weakness in one patient while another patient had asymmetric muscle weakness and rest of the patients had normal muscle power. None of the patient had autonomic dysfunction (Fig-1).

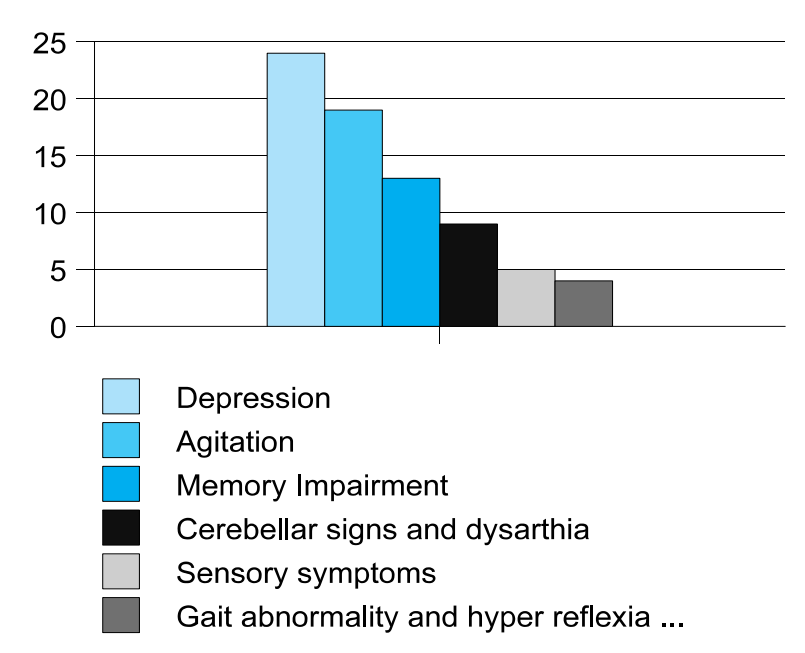

Fig-1. Six most common neuropsychiatric problems
In six neuropsychiatric problems were commonly seen in vitamin B 12 deficient people. In these patients depression was observed in $16 \%$, agitation $12.7 \%$, memory impairment $8.7 \%$, cerebellar signs and dysarthria $6 \%$, sensory symptoms $3.3 \%$ and gait abnormality and hyperreflexia and extensor planters $2.7 \%$.

Radiological examination of Brain was carried out in 29 patients (19.3\%), 18 patients (62\%) of these patients showed abnormal radiological findings. Atrophy was found in $6(4 \%)$, gray matter involvement in $3(2 \%)$, white matter involvement in $8(5.3 \%)$, while basal ganglia was involved in $1(0.7 \%)$ of the patients (Fig2).

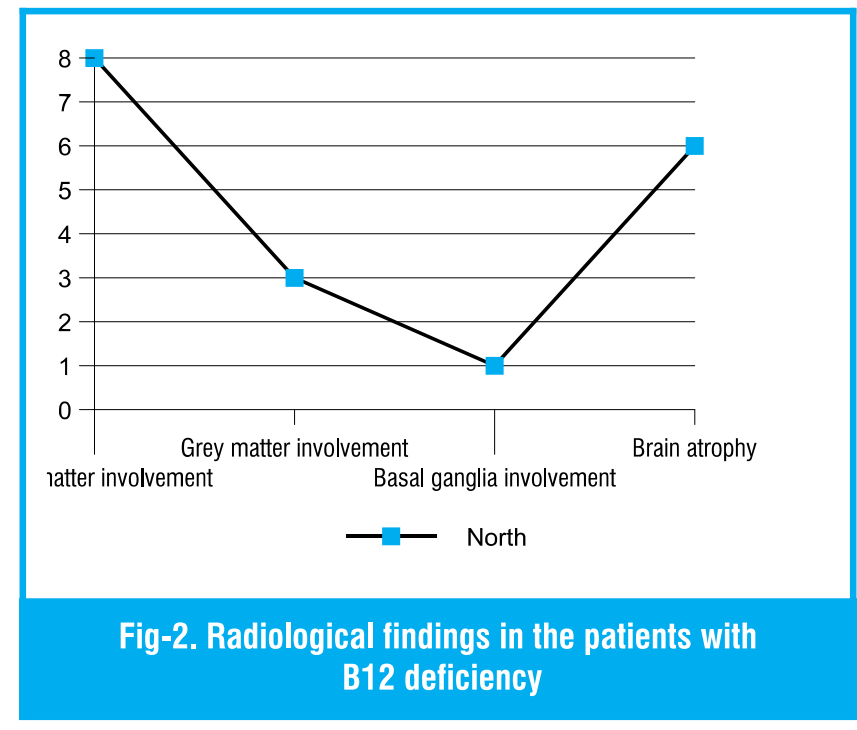

Electro diagnostic study (NCV/ EMG) was done in 13 $(8.7 \%)$ of the patients, in which peripheral neuropathy was recorded in 4 (2.7\%), one patient had entrapment neuropathy, while it was found to be normal in $8(5.3 \%)$ of the patients.

\section{DISCUSSION}

Vitamin B12 deficiency causes diverse neuropsychiatric syndromes along with other systemic manifestations. None of the manifestations are unique to this disease and vitamin B12 deficiency alone is the sufficient cause of neuropsychiatric symptoms. There 
is no age specification and both sexes may be involved. In this study female to male ratio of 1.1:1, as in Caucasian, Hispanic and Asian populations, there is an overall younger age distribution, especially among women'.

Comparatively Indian studies showed a male predominance. In our part of the world probably female patients seek medical attention only when the symptoms get extremely worse. Other western studies also showed that about $5-10 \%$ of persons over the age of 65 years are vitamin b12 deficient which is again not matching with our study ${ }^{10,11}$.

Interestingly in our study we observed that in the age group between $40-59$ years of age there is marked rise in the number of female patients (43 patients) with severe 12 deficiency. This is not observed in other age groups and in male patients.

The subtle difference in the number of female patients as compared to the male patients may be due to the fact that females in our social setups and in our part of the world are more nutritionally deprived as compared to the males. However considering gender distribution, male and female ratio has no marked gross difference throughout the world as in our study.

In our study we have found that 140 patients (93.3\%) belonged to non vegetarians group while 10 patients $(6.7 \%)$ were on vegetarian diet, while the western study showed that the deficiency was more frequent in pure vegetarians than in non vegetarians. Non vegetarians with a meat intake of less than 5 times a month had more frequent deficiency than those with more frequent meat intake. All the cases described by Dastur et al and Jeejeebhoy et al were vegetarians ${ }^{12,13}$.

The probable difference in the results of our study as compared to the western and fore mentioned Indian studies might be due to the fact that most of our studied patients (140 out of 150) were Muslims and consuming meat more in comparison to the western world and India. However we didn't collected data of the fortified cereal intake.

In our study we have found $24(16 \%)$ of the patients had depression and was the leading top most presenting manifestation of low serum B12. Most of the other conducted studies in this perspective have also studied decrease in the Folate levels concomitantly with B12 deficiency ${ }^{14}$. While in our study we have studied the association of depression with low serum B12 levels only.

Memory was impaired in $13(8.7 \%)$ of the patients. It was the second most common neuropsychiatric manifestation studied in our patients. The study of Healton et $\mathrm{al}^{15}$, done in 1991 comprising of 143 patients, showed that the neuropsychiatric manifestations such as recent memory loss with reduced attention span and other wise normal cognition, depression, hypomania, paranoid psychosis with auditory or visual hallucinations (megaloblastic madness), violent behaviour, personality changes, blunted affect and emotional lability were present in $8 \%$ of the studied population. While in our studied population the chief neuropsychiatric manifestations were depression, Agitation, labile mood and memory loss. Similarly the study of Shorvon et $\mathrm{al}^{16}$ showed that the cognitive and behavioral signs were present in $10 \%$ of his studied patients. Hallucinations, paranoid symptoms and hypomania were not found in our studied patients.

Most of the cranial nerve examination did not reveal any significant abnormality. Anosmia was found in 2 patients (1.3\%) and upper motor neuron facial weakness was noted in one $(0.66 \%)$ patient, but the patient had concomitant sub cortical stroke so the exact association between the cranial nerve defect and B12 deficiency cannot be established.

Indian study was carried out in 167 patients; 
neuropathy was detected in 42 cases (25.14\%). Half of these had only sensory neuropathy ${ }^{17}$. As compared to this study we have found glove and stocking sensory loss in 4 (2.7\%) patient, 1 (0.7\%) had dermatomal loss . Western studies have found more frequent neuropathy in B12 deficiency (40\%).

In western studies evidence of spinal cord involvement was found in 12 (7.2\%), and 7 of these had evidence of involvement of peripheral nerves, posterior columns and pyramidal tracts, While in our study $2(1.3 \%)$ of patients had pyramidal symptoms. In the western studies, MRI scan showed cerebellar and cerebral atrophy and defects in myelination. They have found that this atrophy seen on MRI may regress with $B 12$ treatment but the developmental delay may persist ${ }^{18,19}$. While in our study atrophy was found in 6(4\%), grey matter involvement in $3(2 \%)$, white matter involvement was found in $8(5.3 \%)$, while basal ganglia was involved in $1(0.7 \%)$ of the patients. Therefore early detection of vitamin B12 deficiency may help to prevent the developmental deficits associated with B12 deficiency.

\section{CONCLUSIONS}

It was identified through the study that various neuropsychiatry problems (like depression, agitation, and memory problems, gait and cerebellar involvement) are associated with vitamin B12 deficiency.

Therefore it is recommended that when neuropsychiatric patient is encountered, the clinician should not forget to perform vitamin B 12 level, especially with above mentioned problems.

\section{Copyright@ 29 Apr, 2013.}

\section{REFERENCES}

1. Hvas AM, Nexo E. Diagnosis and treatment of vitamin B12 deficiency. An update. Haematologica 2006;91: 1506-12.

2. Wolters M, Strohle A, Hahn A. Cobalamin: a critical vitamin in the elderly. Prev Med. 2004;39(6): 1256-1266.

3. Wolters M, Strohle A, Hahn A. Age-associated changes in the metabolism of vitamin $B(12)$ and folic acid: prevalence, aetiopathogenesis and pathophysiological consequences. Z Gerontol Geriatr 2004;37(2):109-135.

4. Andres E, Federici L, Affenberger S, Vidal-Alaball J, Loukili NH, Zimmer J, et al. B12 deficiency: a look beyond pernicious anemia. J Fam Pract 2007;56:53742.

5. Stanger 0, Fowler B, Pietrzik K, Huemer M, Becher EH, Semmler A, et al. Homocysteine, folate and vitamin B12 in neuropsychiatric diseases: review and treatment recommendations. Expert Rev. Neurother.2009; 9(9):1393-1412.

6. Obeid R, Herrmann W. Mechanisms of homocysteine neurotoxicity in neurodegenerative diseases with special reference to dementia. FEBS Lett.2006; 580(13):2994-3005.

7. $\quad \mathrm{Ho} \mathrm{Pl}$, Ashline D, Dhitavat S. Folate deprivation induces neurodegeneration: roles of oxidative stress and increased homocysteine. Neurobiol. Dis.2003; 14(1):32-42.

8. Carmel $R$, Sarrai M. Diagnosis and management of clinical and subclinical cobalamin deficiency: advances and controversies. Curr Hematol Rep 2006;5:23-33.

9. Katsaros K, Glocker FX. Hemmer B. MRI of spinal cord and brain lesions in sub acute combined degeneration. 1998; 40:716-19.

10. Carethers M. Diagnosing vitamin B12 deficiency: A common geriatric disorder. Geriatics 1988;43:89-94.

11. Marcus DL, Shadick N, Crantz J, Gray M, Hernandenz F, Freedman subpopulation. J Am Geriatr Soc 1987;35:635-8.

12. Dastur DK, Quadros EV, Wadia NH, Desai MM. Effect of vegetarianism and smoking on vitamin B12, thiocyanate, and folate levels in the blood of normal subjects. Br Med J 1972;3:260-3. 
13. Jeejeebhoy KN, Wadia NH, Desai HG. Role of vitamin B12 deficiency in tropical nutritional neuromyelopathy. J Neurol Neurosurg Psychiatry1967;30:7-13.

14. Agarwal R. Vitamin B12 deficiency \& cognitive impairment in elderly population. Indian $\mathrm{J}$ Med Res. 2011 October; 134(4): 410-412.

15. Shorvon SD, Carney MWP, Chanarin I. The

\section{AUTHOR(S):}

1. DR. QAMAR-UN-NISA

MBBS, FCPS

Consultant Nurologist Rabia Moon Hospital

F-13/6 F.C. Area Karachi.

2. DR. MUHAMMAD TANVEER ALAM

MBBS, FCPS

Assistant Prof. of Medicine DUHS \& CHK

A-7 Block. !3-D Gulshan-e- Iqbal Karachi

3. DR. HARILAL KHIEMANI

Assistant Prof. of Medicine DUHS \& CHK

4. Dr. Ayesha Chandna

MBBS, FCPS

Consultant Physician LNH neuropsychiatry of megaloblastic anemia. $B M$ 1980; 281: 1036-38.

16. Lovblad K, Ramelli G, Remonda L. Retardation of myelination due to dietary vitamin B12 deficiency: Cranial MRI findings. Pediatr Radiol 1997; 27:155-8.

17. Wadia RS, Bandishti B, Kharche M. B12 and folate deficiency: incidence and clinical features. Neurol India 2000;48:302-4.
5. Dr. Muhammad Wasay MBBS, MD

Associate Prof. of Neurology AKUH

Correspondence Address:

Dr. Qamar-un-Nisa

MBBS, FCPS

Consultant Nurologist Rabia Moon Hospital

F-13/6 F.C. Area Karachi.

dr_qamar_mukhtar@hotmail.com

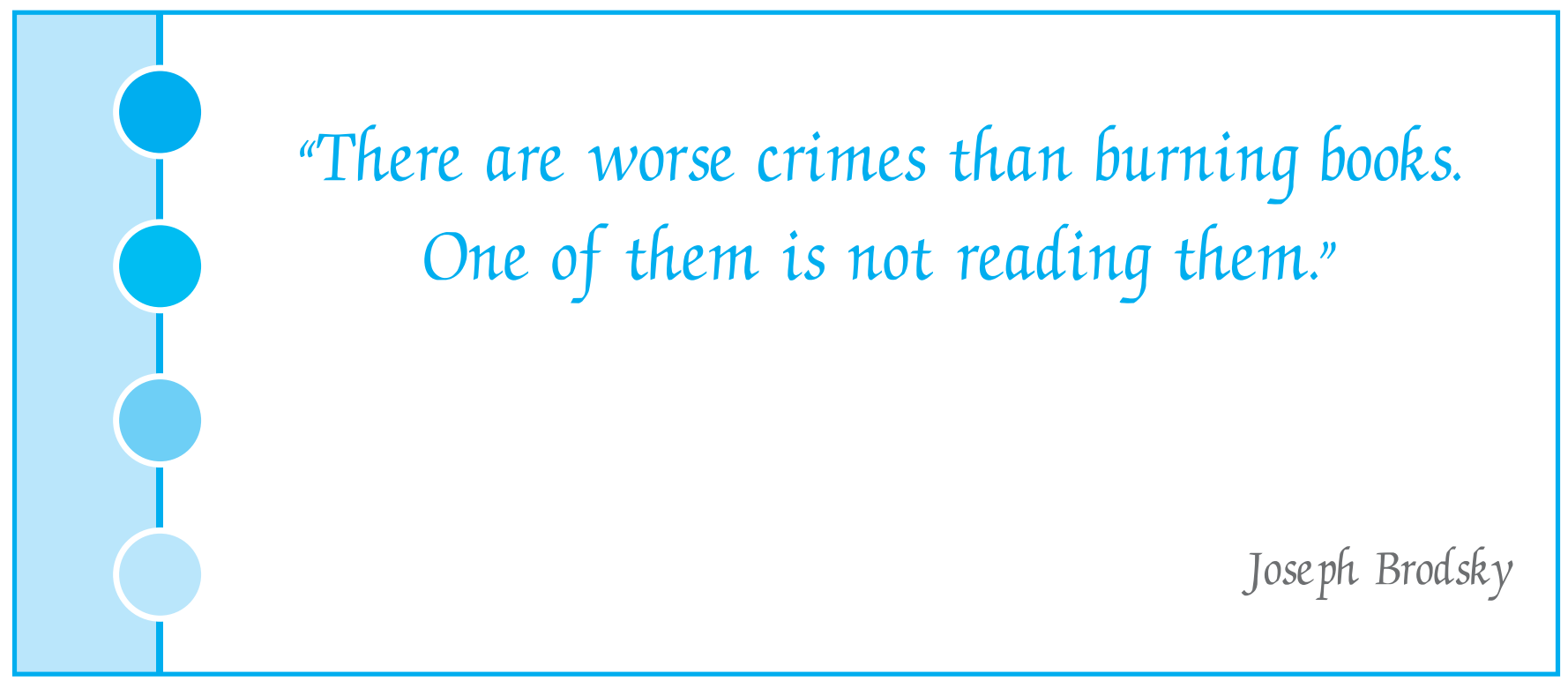

